In zahlreichen Untersuchungen an Eiern und eben ausschlüpfenden Hühnchen konnte ich ScHönHEIMER voll bestätigen, daß das Dihydrosterin tatsächlich intermediären Prozessen seinen Ursprung verdankt. Nach der Bebrütung beobachtete ich eine regelmäßige Vermehrung des hydrierten Sterinanteils, im Durchschnitt von 3,2\% auf 9,1\%. Es soll hier nicht erörtert werden, ob mit dieser Zunahme die gleichzeitig von mir festgestellte, allerdings nicht regelmäßige Vermehrung der Gesamtsterine (um etwa $5 \%$ ) während der Bebrütung im Zusammenhang steht. (THANNHAUSER hatte schon früher, im Gegensatz zu Beumer, Windaus, Gardner eine geringe $Z$ unahme des Gesamtcholesterins gefunden. Diese Frage wird sich nur an einem sehr großen Material klären lassen.)

Dagegen konnte ich in weiteren Versuchen den Nachweis einer gleichzeitigen Dehydrierung zu Ergosterin (Provitamin D) nicht erbringen. Der Ergosteringehalt des Digitoninfällbaren betrug in 3 Eiern $0,17 \%$ und in 3 Hühnchen $0,20 \%$. Der geringe Unterschied liegt in der methodischen Fehlergrenze und innerhalb der biologischen Schwankungsbreite. Die Aufarbeitung erfolgte besonders versichtig und unter Berücksichtigung der Feststellungen v. GOTTBERGs ${ }^{4}$ beim Umkrystallisieren von Cholesterin-Ergosteringemischen.

Die Ergosterinbestimmung wurde spektrophotometrisch durch Herrn Pallutz vom Chemischen Institut ausgeführt, dem ich dafür zu Dank verpflichtet bin.

Zusammenfassung: Das Dihydrocholesterin vervielfacht sich im Hühnerei während der Bebrütung. Eine gleichzeitige Dehydrierung anderen Sterins im Sinne einer Ergosterinvermehrung kann nicht beobachtet werden.

Eine ausführliche Angabe der Versuche erfolgt an anderer Stelle. (Aus der Universitäts-Kinderklinik, Göttingen. [Direktor: Prof. Dr. Beumer].)

Literatur: ${ }^{1}$ Hoppe-Seylers Z. 1929/30. - ${ }^{2} \mathrm{~J}$. of biol. Chem. I929/30. $-{ }^{3}$ Naturwiss. I930, H. 42 . $-{ }^{4}$ Dissertation Göttingen I930.

\section{ÜBER DIE WIRKUNG DES HYPOPHYSINS AUF DEN WASSERSTOFFWECHSEL.}

Von

P. RoBoz.

Der Hypophysenhinterlappenextrakt übt mehrere Wirkungen auf den Wasserstoffwechsel aus. Die für den Kliniker wichtigste und am besten bekannte Wirkung ist die Diuresehemmung nach Wasserbelastung und ihre therapeutische Anwendung beim Diabetes insipidus. Zur Erklärung der Diwresehemmung wurde einerseits auf eine, noch bisher nicht einwandfrei festgestellte Gewebswirkung im Sinne einer gesteigerten Wasserbindungsfähigkeit hingewiesen (VEILE. MEYER und R. MEYER-BISCH u.a.), andererseits eine Nierenwirkung betont (Literatur s. bei TRENDELENBURG, ElLINGER und Poulsson).

Es ist ferner bekannt, daß der Hypophysenhinterlappenextrakt, abhängig von Tierart (HousSAY), Dosis (KonschegG und Schuster), Narkose (MAgnus und SchÄFER usw.), Ort der Einspritzung (FromHERz), die Urinausscheidung sowohl hemmend wie fördernd beeinflussen kann. Zur Erklärung dieser Wirkungen wurde auch angenommen, daß der Hypophysenhinterlappenextrakt eine diuresehemmende und eine diuresefördernde Phase besitzt. (Fromherz). Um die umstrittene Frage zu prüfen, ob die diuresehemmende Wirkung des Hypophysins nur durch einen renalen Angriffspunkt bedingt ist oder, wie es die anderen genannten Forscher annehmen, auch die gesteigerte Wasserbindungsfähigkeit der Gewebe darin eine wichtige Rolle spielt, wurden Versuche mit folgender Versuchsanordnung vorgenommen. Es wurde in einer ersten Versuchsreihe bei Kaninchen Hypophysin "Höchst" subcutan gespritzt und seine Wirkung auf den Blutwassergehalt und die Urinausscheidung festgestellt. In einer zweiten Versuchsreihe wurde bei denselben Tieren $50 \mathrm{ccm}$ körperwarme Ringerlösung infundiert die Blut- verdünnung mittels Hämoglobinbestimmungen und der Einfluß der Ringerlösung auf die Diurese bestimmt. In einer dritten Versuchsreihe wurde unter denselben Versuchsbedingungen die Ringerinfusion nach der Einspritzung des Hypophysins vorgenommen.

Diese Versuche erwiesen zunächst die schon wiederholt gefundene (Modrakowski und Halter, Mrura u. a.) Hydrämiewirkung des Hypophysenextraktes. Im einzelnen. ließ sich dabei folgendes feststellen:

Nach kleineren Dosen Hypophysin tritt eine Diureseförderung, nach größeren Dosen meistens eine Diuresehemmung auf, und in einer zweiten Periode kann unter Umständen auch nach gróßen Dosen eine Diureseförderung auftreten. Die Hydrämie ist bei den kleinen diuresefördernden Dosen gering, bei den großen Dosen hochgradig. Es konnte beobachtet werden, daß das Hypophysin die Urinausscheidung nach Ringerinfusion hemmt und da $B$ die Blutverdünnung nach Hypophysineinspritzung und Ringerinfusion stärker ist als nach Ringerinfusion allein. Mit besonderer Klarheit konnte die Differenz zwischen Ringerhydrämie und der Hydrämie, die nach Ringerinfusion und Hypophysineinspritzung auftritt, dann beobachtet werden, wenn so kleine Hypophysindosen verabreicht wurden, die selbst nur eine geringe oder gar keine Hydrämie verursachten.

Diese Versuchsergebnisse sprechen dafür, daß durch das Hypophysin die Wasserbindungsfähigkeit der Gewebe nicht gesteigert wird und die diuresehemmende Wirkung des Hypophysins nur durch eine Nierenwirkung bedingt ist.

Es konnte weiter gezeigt werden, daß bei der Entstehung der Hypophysinhydrämie nicht nur die durch Diuresehemmung bedingte Wasserretention, sondern auch eine Gewebswassermobilisation beteiligt ist. Dafür, daß das Hypophysin nicht nur eine nierensperrende, sondern auch eine gewebswassermobilisierende Wirkung hat, sprechen erstens die Versuche von Mrura bei nephrektomierten Tieren, zweitens der Umstand, da $\beta$ auch bei den kleinen diuresefördernden Dosen eine Hydrämie auftritt, und drittens der Nachweis, daß die durch Diuresehemmung bedingte Wasserretention im allgemeinen nicht die hochgradige Hydrämie rechnerisch erklären kann.

Es wurde auf Grund dieser Ergebnisse darauf hingewiesen, $\mathrm{da} B$ in der Entstehung der sowohl nach den großen, als auch nach den kleinen Dosen auftretenden Diureseförderung die Wassermobilisation aus den Geweben eine wichtige Rolle spielt. Es wird in meiner demnächst im Arch. f. exper. Path. erscheinenden ausführlichen Arbeit ferner die Auffassung begründet, daß die Wirkung des Hypophysins auf die Diurese einphasisch ist. Die nach den großen Dosen in der zweiten Periode auftretende Diureseförderung ist eine sekundär bedingte und wird durch die Wasserverschiebung im Organismus in der ersten Phase verursacht, die einerseits durch die Diuresehemmung, andererseits durch die Gewebswassermobilisation hervorgerufen wird. (Aus der Kieler UniversitätsKinderklinik [Vorstand: Prof. Rominger].)

\section{ÜBER DEN EINFLUSS DER MILZ AUF DAS MORPHO- LOGISCHE BLUTBILD. \\ Von \\ A. W. Selesnjew.}

Der Einfluß der Milz auf das morphologische Bild des Blutes wurde in $3^{\circ}$ Versuchen an 29 Hunden untersucht. Das Blut für die Untersuchung wurde aus verschiedenen Teilen des Venensystems und der Milzarterie entnommen. Als wirkende Agenzien wurden Adrenalin, Bariumchlorid, Atropin und Amylnitrit angewandt. Ein Teil der Versuche wurde, ohne Anwendung irgendeines Agens, als Kontrolle angestellt.

Die geformten Elemente des Blutes sind in den Gefäßen der gesunden Tiere gleichmäßig verteilt, bei einem konstanten Verhältnis der einzelnen Formen untereinander. Adrenalin ruft eine ungleichmäßige Verteilung der weißen Blutkörper- 